\title{
B-type natriuretic peptide (NT-proBNP) and thyroid function: study in elderly subjects
}

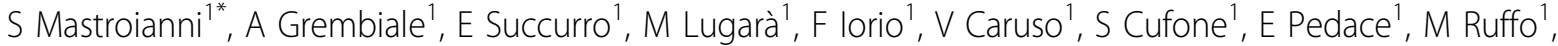 \\ C Cloro $^{2}$, F Arturi $^{1}$
}

From de Senectute: Age and Health Forum

Catanzaro, Italy. 5-7 December 2009

\section{Background}

B-type natriuretic peptide (NT-proBNP) is a cardiac neurohormone that is secreted from the left ventricle in response to volume and pressure overload as occurs in patients with congestive heart failure. NT-proBNP has now become a useful tool in the diagnosis of congestive heart failure and in the management of patients with acute dyspnea. In recent years, several studies showed that NT-proBNP levels may be influenced by thyroid diseases. However, there aren't data on the elderly population, a group of patients in whom the incidence of both thyroid diseases and heart failure is frequent. The aim of this study was to assess the levels of NT-proBNP in a group of elderly patients with dyspnea and thyroid disease and without congestive heart failure.

\section{Materials and methods}

We examined 41 patients with thyroid disease: 10 with overt hyperthyroidism, 8 with subclinical hyperthyroidism, 8 with subclinical hypothyroidism, 7 with overt hypothyroidism and 8 with euthyroid multinodular or uninodular goiter. As a control, we used 30 patients without thyroid disease or other conditions that could influence the levels of NT-proBNP. Serum levels of NTproBNP, thyroid hormones, echocardiographic parameters and Holter ECG were evaluated in all subjects. In patients submitted to antithyroid or replacement therapy, the valuation of serum levels of NT-proBNP was performed before and after treatment.

\section{Results}

NT-proBNP levels were significantly higher in patients with overt hyperthyroidism and subclinical hyperthyroidism compared with control subjects and patients with normal thyroid function. NT-proBNP levels were lower in patients with overt hypothyroidism compared to normal subjects. Conversely, there was no significant difference between the levels observed in patients with subclinical hypothyroidism and those of normal subjects. Drug treatment was able to induce a normalization of NT-proBNP levels both in patients with hyperthyroidism and in patients with hypothyroidism.

\section{Conclusions}

The levels of NT-proBNP are influenced by a thyroid function with a more pronounced effect of hyperthyroidism than hypothyroidism. Therefore in the presence of elevated values of NT-proBNP and the absence of signs of heart failure it is necessary to consider the possible presence of a condition of overt or subclinical hyperthyroidism.

\section{Author details}

'Dipartimento di Medicina Sperimentale e Clinica, Università degli Studi "Magna Graecia"di Catanzaro, Italy. ${ }^{2}$ Unità Operativa di Cardiologia, Ospedale Civile"SS Annunziata" di Cosenza, Italy.

Published: 19 May 2010

\section{doi:10.1186/1471-2318-10-S1-A85}

Cite this article as: Mastroianni et al:: B-type natriuretic peptide (NTproBNP) and thyroid function: study in elderly subjects. BMC Geriatrics 2010 10(Suppl 1):A85. 